UCRL-JC-127860

PREPRINT

\title{
Chemical Identity of Atoms Using Core Electron Annihilations
}

\author{
P. Asoka-Kumar
}

This paper was prepared for submittal to International Conference on Positron Annihilation

Kansas City, MO

May 25-30, 1997

June 27, 1997

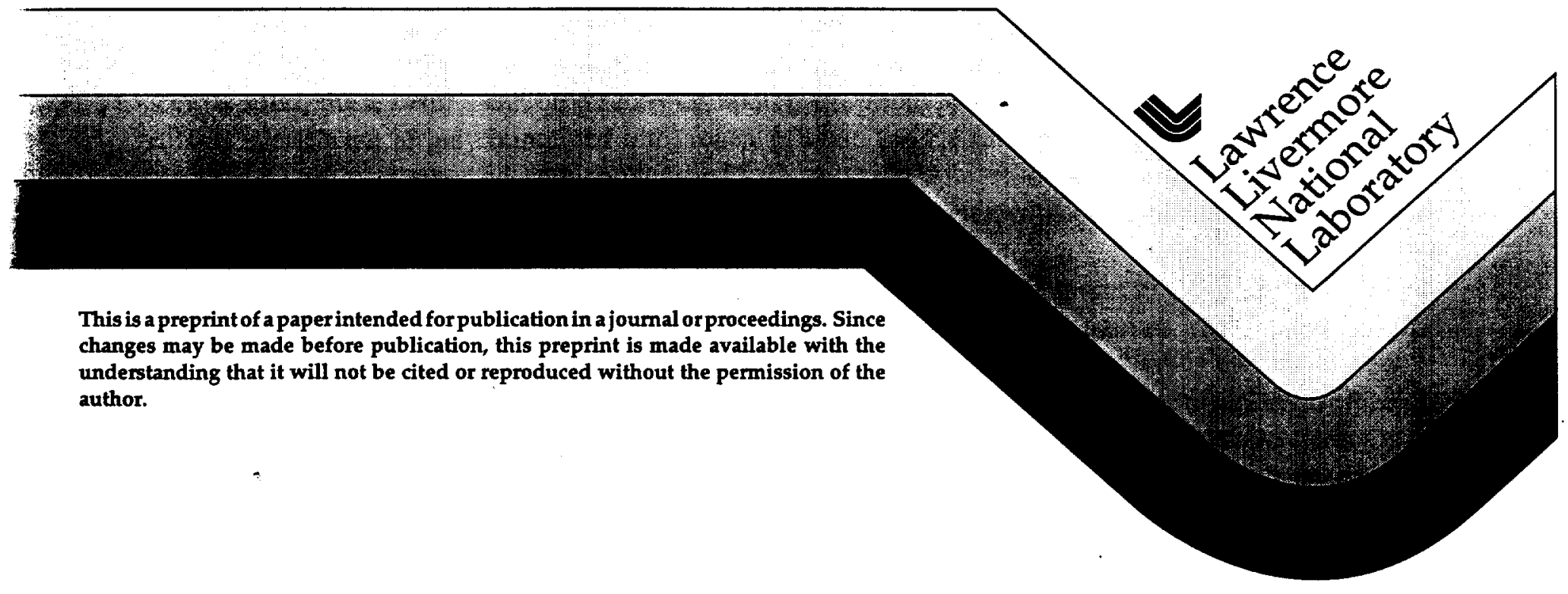




\section{DISCLAIMER}

This document was prepared as an account of work sponsored by an agency of the United States Government. Neither the United States Government nor the University of California nor any of their employees, makes any warranty, express or implied, or assumes any legal liability or responsibility for the accuracy, completeness, or usefulness of any information, apparatus, product, or process

disclosed, or represents that its use would not infringe privately owned rights. Reference herein to any specific commercial product, process, or service by trade name, trademark, manufacturer, or otherwise, does not necessarily constitute or imply its endorsement, recommendation, or favoring by the United States Government or the University of California. The views and opinions of authors expressed herein do not necessarily state or reflect those of the United States Government or the University of California, and shall not be used for advertising or product endorsement purposes. 


\title{
Chemical identity of atoms using core electron annihilations
}

\author{
P. Asoka-Kumar \\ Lawrence Livermore National Laboratory, Livermore, CA 94550
}

Keywords: Elemental specificity, chemical identification, high-momentum electrons

\begin{abstract}
Positron annihilation spectroscopy is a sensitive probe for studying the electronic structure of defects in solids. The high momentum part of the Doppler-broadened annihilation spectra can be used to distinguish different elements. This is achieved by using a new two-detector coincidence system and by imposing appropriate kinematic cuts to exclude background events. The new setup improves the peak to background ratio in the annihilation spectrum to $\sim 10^{5}$. As a result, the line shape variations arising from different core electrons can be studied. The new approach adds elemental specificity to the Doppler broadening technique, and is useful in studying elemental variations around a defect site. Results from several case studies are reviewed.
\end{abstract}

\section{Introduction}

The Doppler broadening of the annihilation gamma rays has been used extensively to study vacancies and vacancy clusters[1]. Despite the remarkable success of this observable in rapidly identifying dilute quantities of defects, the Doppler broadening parameters $(\mathrm{S}, \mathrm{W}, \mathrm{etc}$.) have been difficult to calculate from the electron momentum distribution. Due to this difficulty, many in the positron community have shied away from the Doppler broadening measurements and have concentrated on theoretically verifiable observables (lifetime and angular correlation). In this paper, I show the recent improvements in Doppler broadening measurements that is making it an equally attractive positron tool.

Nearly two decades ago, Lynn et al. showed how the Doppler broadening measurements can yield the momentum profiles of core electrons[2,3]. This is achieved by replacing the standard single $\mathrm{Ge}(\mathrm{Li})$ detector with a coincident two $\mathrm{Ge}(\mathrm{Li})$ detector setup and by imposing appropriate kinematic cuts to the resulting spectrum[4]. Because the core electrons retain their atomic character even when atoms form a solid, these results can be directly compared to simple theoretical predictions. The importance of this work went largely unnoticed until recently when the Helsinki group started applying them to study defects in compound semiconductors[5,6]. A systematic study of the line shape variations originating from the high momentum electrons of different elements demonstrated the elemental specificity of this new approach[7].

The conventional Doppler broadening spectra obtained by recording one of the photons from the annihilation process is distorted by background events (high energy gamma rays, pulse pileup, incomplete charge collection, and three-photon decay of positronium). This problem is particularly acute in the energy region (tail regions of the annihilation spectrum) where the contributions from high momentum core electrons are dominant. As discussed by Saarinen here, a single Ge detector in coincidence with a timing detector ( $\mathrm{NaI}$ or $\mathrm{BGO}$ ) will reduce the background on the high-energy side of the annihilation peak [8]. The two-photon coincidence measurement provides a more informative kinematic picture of the annihilation process.

\section{Two-photon Doppler system}

The principle of the two-photon Doppler system is described elsewhere[4,7]. With the simultaneous recording of the energies of both gamma rays $\left(\mathrm{E}_{1} \& \mathrm{E}_{2}\right)$, events meeting the total energy criteria of $E_{T}=E_{1}+E_{2}=2 m_{0} c^{2}-E_{b}$ can be selected to suppress the background events, where $E_{b}$ is the 


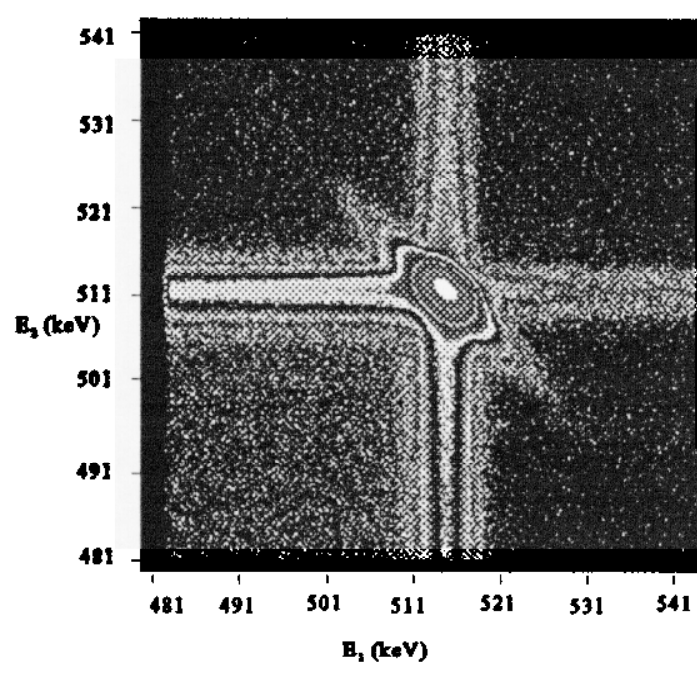

Figure 1 A two-dimensional display of coincidence spectrum

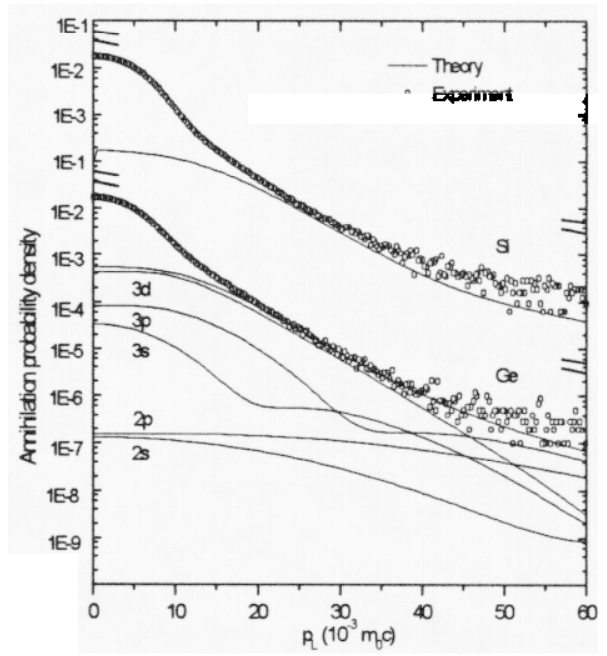

Figure 2 Annihilation probability density for $\mathrm{Si}$ and $\mathrm{Ge}$ as a function of the logitudinal momentum. $\mathrm{p}_{\mathrm{L}}$.

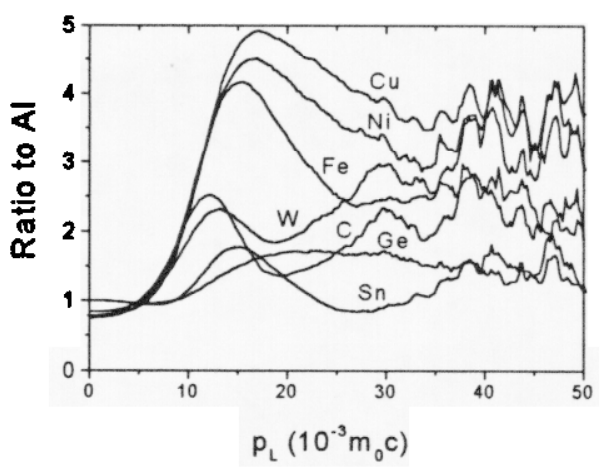

electron binding energy. The energy difference, $E_{1}-E_{2}$, is equal to $p_{L} c$, where $R$ is the momentum of the electronpositron system along the direction of the gamma ray emission. Figure 1 shows an example of a two-dimensional spectrum. The gray tone indicates the number of counts. The central peak at $E_{1}=E_{2} \approx m_{0} c^{2}$ corresponds to annihilations with small Doppler shifts. The horizontal and vertical bands extending from the central peak are produced by coincidences of a background photon with a $511 \mathrm{keV}$ photon. The elliptical region extending diagonally with $E_{1}+E_{2}-2 m_{0} c^{2}=1022 \mathrm{keV}$ originates from the high momentum electrons, and this region is nearly background free. Therefore, a cross section along the diagonal can be analyzed to study the core-electron momentum distributions.

\section{Elemental Specificity}

Figure 2 shows the shape variations arising from core annihilations of $\mathrm{Si}$ and $\mathrm{Ge}$. The experimental curves are obtained by folding the Doppler-broadening curve and normalizing to obtain the annihilation probability density as a function of the longitudinal momentum. The details of the normalization procedure can be found in Ref. 7. The samples used in the present study are high-purity single crystals, and are "defect-free" as seen by positrons. The spectra were collected at an incident positron beam energy of $30 \mathrm{keV}$ to exclude positron diffusion to the surface and subsequent annihilations from surface states. The larger amplitude of the Ge curve stems from the fact that the outermost core shell in the case of $\mathrm{Ge}$ is $3 \mathrm{~d}$, which is highly occupied and spatially extended. The theoretical curves are obtained using the momentum distribution for each core electron state within the independent particle model, using free atom wave functions[9]. The contributions from the individual shells of Ge are also included. Since the core electrons retain their atomic character even when atoms form a solid, atomic wave functions provide an accurate description of the core electron states. Atomic wave functions, however, are not an accurate description of the valence electrons and are not added to the total.

Since the curves in Fig. 2 span several orders of magnitude, the shape difference between them can be seen more easily after normalizing the measured spectrum to a reference spectrum. Fig. 3 shows the ratio curves for several elements and demonstrates the elemental specificity of the new method.

\section{Vacancies and impurity-vacancy complexes}

Figure 3 Ratio of the annihilation probability densities of different elements to $\mathrm{Al}$. 


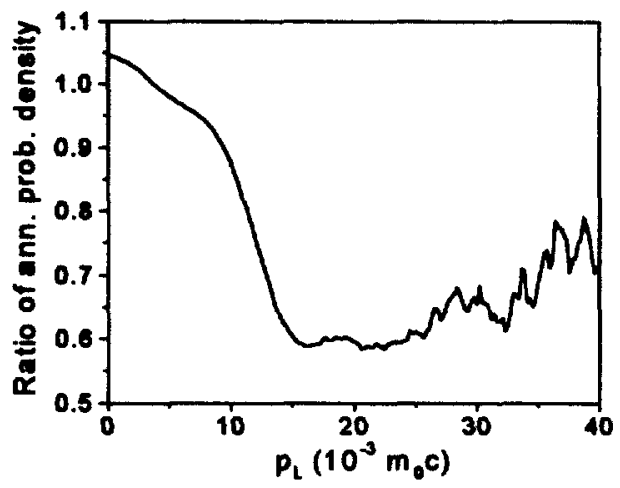

Figure 4 Ratio of annihilation probability density for thermally generated vacancies in Al.

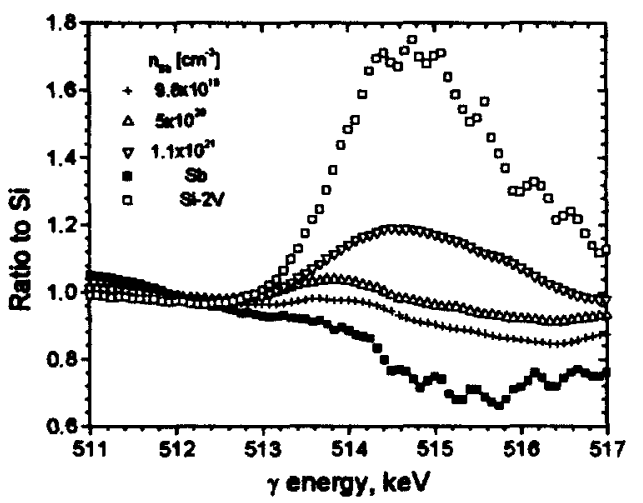

Figure 5 Ratio curves for $\mathrm{Sb}$ doped $\mathrm{Si}$ as a function of gamma ray energies. Results for Si divacancies are also shown (after ref. 10).

about $\mathrm{N}_{\mathrm{Sb}}=7 \times 10^{20} \mathrm{~cm}^{-3}$, and further increase in dopant density does not show a corresponding increase in the carrier concentration (for details see Ref. 10).

The core annihilation line shape clearly shows the presence of Sb for concentrations beyond the critical dopant density. When $\mathrm{Sb}$ occupies a lattice site in $\mathrm{Si}$ and is activated, the $\mathrm{Sb}$ ion is positively charged. Therefore, positrons cannot sense them. Figure 5 shows the ratio curves at three Sb concentrations along with the results from bulk $\mathrm{Sb}$. The Sb signal is clearly evident at the highest dopant concentration. Since positrons are not sensitive to ionized $\mathrm{Sb}$ atoms, the presence of Sb signal at the high dopant concentration can be understood in terms of a Sb-vacancy complex. Modeling of the shape variations show that the data points are consistent with a linear superposition of Si vacancy signal and $\mathrm{Sb}$ signal[10]. The fraction of the $\mathrm{Sb}$ signal required is consistent with the observed reduction in carrier concentration. Since Sb-vacancy complex is an acceptor, it can compensate the substitutional Sb donors.

Ion implantation is routinely used in device fabrication to introduce controlled quantities of dopant atoms in isolated regions of a semiconductor wafer, thus transcending the limits set by thermal diffusion. The ion implantation, besides introducing foreign atoms, creates many lattice defects in the solid. Several impurity-vacancy related problems are present in ion implantation. When the implantation dose exceeds solid solubility, the precipitation can become severe. The implantation damage can act as gettering centers. The oxygen-vacancy complex can be formed in Czochralski Si. The contamination of the primary beam from the residual gas scattering can occur in high energy implantations using multiply 


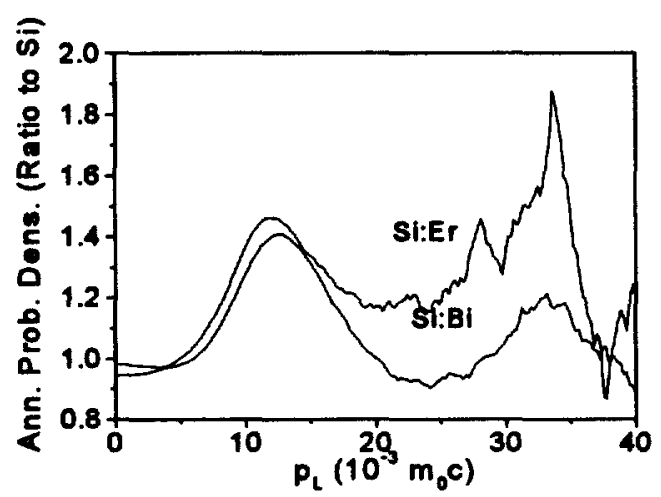

Figure 6Annihilation probabilty density for Er and Bi implanted in $\mathrm{Si}$, after normalizing to bulk $\mathrm{Si}$.

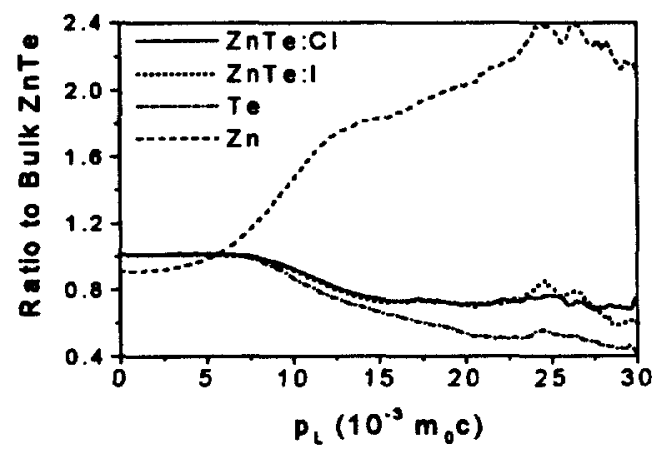

Figure 7Annihilation probability density of ZnTe films doped with $\mathrm{Cl}$ and I. Results for bulk $\mathrm{Zn}$ and Te are also shown. The data is normalized to a $\mathrm{ZnTe}$ polycrystal showing a single lifetime value of 286 ps.

understood as arising due to the first neighbor of a $\mathrm{Zn}$ vacancy (see Figure 7).

\section{Novel studies using the coincidence method}

Before concluding, I also wanted to point out few novel studies that one could undertake using the two-photon coincidence method. Positronium (Ps) formation can easily be detected using the twodimensional spectra. Ps formation adds two new features to the spectrum shown in Fig. 1. The central peak narrows due to the two-photon decay of Ps. Also, there will be more counts in the lower-left quadrant $\left(E_{1} \& E_{2}<511 \mathrm{keV}\right)$ from the three photon decay mode of Ps. A more systematic study is needed to take advantage of these new features.

The projection of the two-dimensional spectrum onto $E_{1}-E_{2}$ axis provides the annihilation probability density as a function of the longitudinal momentum $\mathrm{p}_{\mathbf{L}}$. The projected spectrum includes contributions from all electrons. The high momentum part of the spectrum is dominated by the innershell electrons, and the approach described above can detect only the sum contributions of all inner-shell electrons. The annihilation of the inner-shell electrons with positrons will create a core hole, which will be filled with the emission of an Auger electron or X-ray. Therefore, the detection of the characteristic $\mathrm{X}$-ray or Auger electron can be used to gate the two-dimensional spectrum to study the contribution of a specific atomic level. The partial annihilation rate and annihilation probability distribution can then be compared directly to understand the positron overlap with the inner-shell electrons. Positron lifetime 
measurements and the corresponding theoretical values show remarkable agreement. However, the positron lifetime is dominated by outer electron contributions and is insensitive to core contributions. Hence, the measurement of the inner-shell annihilation probability distributions will be valuable in validating the theoretical calculations.

The annihilation of energetic positrons can be used to study the spin-dependent properties of a material. With a polarized positron beam (positron beams derived from a radioactive source retains some amount of polarization) the annihilation-in-flight cross section will depend on the spin alignment of the electrons. The annihilation-in-flight events will have a total energy greater than $1022 \mathrm{keV}$ and will be clearly distinguishable in the upper right quadrant of the two-dimensional spectrum of Fig. 1.

\section{Conclusion}

The recent developments in Doppler broadening technique is exciting and is useful in distinguishing between different elements. Because the core electrons retain their atomic character even when atoms form a solid, their annihilation spectra reflect the atomic character and are elemental specific. The method can be used to study a wide variety of problems, like simple open-volume defects, open-volume defects decorated with foreign elements, and vacancies in different sublattices of a compound material.

This work was performed under the auspices of the U.S. DOE by the Lawrence Livermore National Laboratory under Contract No.W-7405-ENG-48. Most of the work described here was performed at Brookhaven National Laboratory under the U.S. DOE contract DE-AC02-76CH00016. I wish to acknowledge the discussions with M. Alatalo, V.J. Ghosh, R. Howell, A.C. Kruseman, K.G. Lynn, B. Nielsen, and S. Szpala.

\section{References}

1. P.J. Schultz and K.G. Lynn, Rev. Mod. Phys. 60 (1988), p. 701, and references therein.

2. K.G. Lynn, J.R. MacDonald, R.A. Boie, L.C. Feldman, J.D. Gabbe, M.F. Robbins, E. Bonderup, and J. Golovchenko, Phys. Rev. Lett., 38 (1977), p. 241.

3. K.G. Lynn, J.E. Dickman, W.L. Brown, M.L. Robbins, and E. Bonderup; Phys. Rev. B 20 (1979) p. 3566.

4. J.R. MacDonald, K.G. Lynn, R.A. Boie, and M.F. Robbins, Nucl. Instrum. and Meth., 153 (1978), p. 189.

5. M. Alatalo, H. Kauppinen, K. Saarinen, M.J. Puska, J. Makinen, P. Hautojarvi, and R.M. Nieminen, Phys. Rev. B 51 (1995), p. 4176.

6. K. Saarinen, this volume, and references therein.

7. P. Asoka-Kumar, M. Alatalo, V.J. Ghosh, A.C. Kruseman, B. Nielsen, and K.G. Lynn, Phys. Rev. Lett. 77(1996), p. 2097.

8. K.G. Lynn and A.N. Goland, Solid State Commun. 18 (1976), p. 1599.

9. M. Alatalo, B. Barbeiellini, M. Hakala, H. Kauppinen, T. Korhonen, M.J. Puska, K. Saarinen, P. Hautojarvi, and R.M. Nieminen, Phys. Rev. B 54 (1996), p. 2397.

10. S. Szpala, P. Asoka-Kumar, B. Nielsen, J.P. Peng, S. Hayakawa, K.G. Lynn, and H.-J. Gossmann, Phys. Rev. B 54 (1996), p. 4722.

11. D.J. Chadi, Phys. Rev. Lett. 72 (1994), p. 534. 


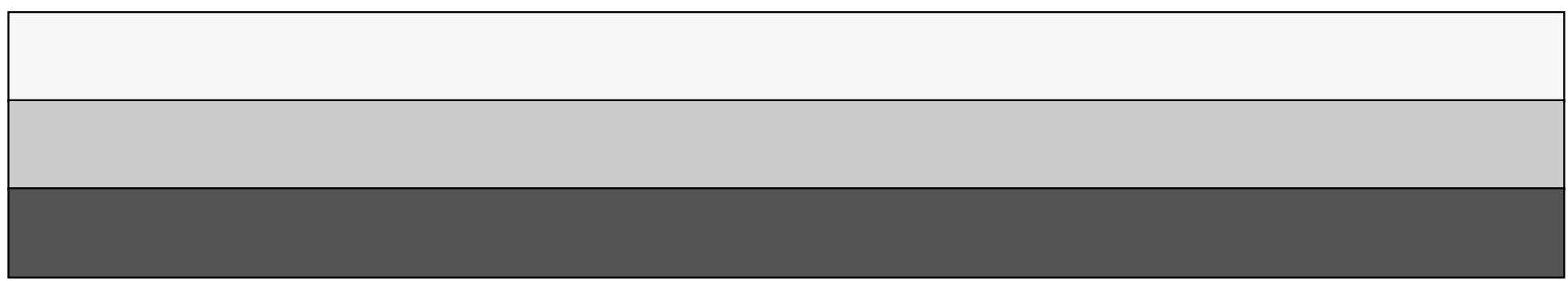

